\title{
Complicações da cardiomiopatia chagásica em paciente adulto jovem
}

\author{
Complications of chagasic cardiomyopathy in young adult patient
}

\author{
Complicaciones de la cardiomiopatía chagásica en paciente adulto joven
}

\begin{abstract}
Ana Flávia Parreira de Morai ${ }^{1 *}$, Dandara Freitas da Silveira e Souza1, Murilo Henrique Fabri Tomazini ${ }^{1}$, Rui Pereira Caparelli de Oliveira ${ }^{1}$.
\end{abstract}

\begin{abstract}
RESUMO
Objetivo: Analisar a evolução da cardiomiopatia chagásica em pacientes adultos jovens, identificando complicações, abordagens diagnósticas e terapêuticas mais atuais. Métodos: Revisão integrativa da literatura nas cinco principais bases de dados disponíveis para pesquisa. Os critérios de inclusão foram os idiomas português, inglês e espanhol; artigos publicados no período de 2010 a 2020, com os descritores: cardiomiopatia chagásica e complicações. Resultados: Foram encontrados 184 artigos e após análise dos critérios de exclusão, foram incluídos nove, sendo eles: caso controle (1), estudos transversais (2), revisões (4), ensaios clínicos randomizados (1) e coorte retrospectivo (1). Os exames diagnósticos como sorologia para Trypanossoma Cruzi, eletrocardiograma e ressonância magnética foram relevantes para o seguimento dos pacientes com cardiomiopatia chagásica. Como opções terapêuticas, o transplante cardíaco e o transplante de células da medula óssea demonstraram resultados satisfatórios; em contrapartida, a terapia medicamentosa com benznidazol não mostrou benefício na cardiopatia chagásica crônica com dano de órgão específico. Considerações finais: Os achados indicam que existem tratamentos que poderiam auxiliar em tais complicações, como o transplante cardíaco e a terapia com células da medula óssea. A escassez de estudos mostra a necessidade de mais pesquisas para desenvolver terapias mais eficazes para uma melhora da sobrevida desses pacientes.
\end{abstract}

Palavras-chave: Cardiomiopatia chagásica, Complicações, Insuficiência cardíaca.

\begin{abstract}
Objective: To analyze the evolution of Chagasic cardiomyopathy in young adult patients, identifying in the literature the main complications and the most current diagnostic and therapeutic approaches. Methods: Integrative literature review in five main databases for research. The inclusion criteria were Portuguese, English and Spanish; articles published between 2010 to 2020, with descriptors chagasic cardiomyopathy and complications. Results: 184 articles were found and nine were included, namely: case control (1), crosssectional studies (2), reviews (4), randomized clinical trials (1) and retrospective cohort (1). Diagnostic exams such as serology for Trypanossoma Cruzi, electrocardiogram and magnetic resonance were relevant for the follow-up of patients with Chagasic Cardiomyopathy. As therapeutic options, heart transplant and bone marrow cell transplant showed suitable results; in contrast, drug therapy with benznidazole did not show any benefit in chronic Chagas Heart Disease with specific organ damage. Final considerations: Findings indicate that there are treatments that could assist in such complications, such as heart transplantation and bone marrow cell therapy. The scarcity of studies shows the need for further researches to develop more effective therapies to improve the survival of these patients.
\end{abstract}

Keywords: Chagasic cardiomyopathy, Complications, Heart failure.

\section{RESUMEN}

Objetivo: Analizar la evolución de la cardiomiopatía chagásica en pacientes adultos jóvenes, identificando en la literatura las principales complicaciones y los enfoques diagnósticos y terapéuticos más actuales. Métodos: Revisión integradora de la literatura en las cinco principales bases de datos disponibles para la investigación. Los criterios de inclusión fueron portugués, inglés y español; artículos publicados entre 2010 y 2020, con los

1 Universidade de Franca (UNIFRAN), Franca - SP. *E-mail: anaflaviapm56@gmail.com 
descriptores: miocardiopatía chagásica y complicaciones. Resultados: Se encontraron 184 artículos y tras analizar los critério de exclusión se incluyeron nueve, a saber: caso control (1), estudios transversales (2), revisiones (4), ensayos clínicos aleatorizados (1) y cohorte retrospectivo (1). Los exámenes diagnósticos como la serología para Trypanossoma Cruzi, el electrocardiograma y la resonancia magnética fueron relevantes para el seguimiento de los pacientes con miocardiopatía chagásica. Como opciones terapéuticas, el trasplante de corazón y el trasplante de células de médula ósea mostraron resultados satisfactorios, en cambio, la farmacoterapia con benznidazol no mostró ningún beneficio en la cardiopatía chagásica crónica con daño orgánico específico. Consideraciones finales: Los hallazgos indican que existen tratamientos que podrían ayudar en tales complicaciones, como el trasplante de corazón y la terapia con células de médula ósea. La escasez de estudios muestra la necesidad de realizar más pesquisas para desarrollar terapias más efectivas para optimizar la supervivencia de estos pacientes.

Palabras clave: Cardiomiopatía de Chagas, Complicaciones, Insuficiencia cardíaca.

\section{INTRODUÇÃO}

A Doença de Chagas é considerada pela Organização Mundial da Saúde (OMS) como uma das doenças infecciosas negligenciadas, com estimativa de atingir mais de oito milhões de pessoas infectadas em todo 0 mundo (SCHIMIDT FILHO A, et al., 2016; BOCHI EA, 2016; SILVA HC, et al., 2013).

De acordo com Guidelines for the diagnosis and treatment of Chagas disease (2019), mesmo em pacientes com sintomas da Doença de Chagas e moradores de regiões endêmicas, a suspeita clínica ou o diagnóstico é muito raro. Tal fato se correlaciona a informações e prática clínica insuficientes entre os medicos e profissionais de saúde sobre este assunto (Diretriz Doença de Chagas 2019 - OMS)

As estimativas mais recentes no Brasil relativas ao número de pessoas infectadas variam de 1,9 milhão a 4,6 milhões de pessoas, cerca de 1,0 a 2,4\% da população (DIAS JC, et al.,2016). De acordo com MartinsMelo FR, et al. (2014), as maiores prevalências são encontradas em mulheres, maiores de 60 anos e residentes nas regiões Nordeste e Sudeste e, segundo Junior ASM, et al. (2018), dentre os pacientes infectados pelo Trypanossoma Cruzi (T. CruzI), cerca de 20\% a 30\% desenvolvem a cardiomiopatia chagásica.

Dentre os diagnósticos mais utilizados atualmente para identificar os infectados pelo T. Cruzi encontra-se: 1) ELISA; 2) Teste imunocromatográfico; 3) Imunoensaio Quimioluminescente de Micropartículas; 4) combinação de dois testes sorológicos positivos (ELISA, ensaio de inibição de hemaglutinação ou imunofluorescência indireta), e potencialmente um terceiro teste, caso os resultados sejam conflitantes (PAHO, 2019).

A histórica clínica da doença de Chagas compreende duas fases distintas: aguda e crônica. A fase aguda se inicia após o período de incubação, o qual pode variar em cerca de um a quatro semanas após contato com o T. Cruzi. A maioria dos pacientes nessa fase são assintomáticos, sendo que complicações como a miocardite ou meningoencefalite assintomática são incomuns nessa fase (SIMÕES MV, et al., 2018).

Apesar da ausência de manifestações clínicas na maioria dos pacientes que se encontram na fase aguda, existe um grupo seleto de menos de $5 \%$ dos pacientes que pode evoluir com a fatalidade devido ao fato de já possuírem insuficiência cardíaca refratária. A fase crônica ocorre após quatro a oito semanas geralmente sendo essa fase dividida em duas formas: indeterminada (latente ou pré-clínica) e determinada, a qual pode ser classificada como cardíaca, digestiva ou cardiodigestiva. Além disso, a forma crônica pode sofrer um processo de reagudização em indivíduos que se encontram em estado de imunossupressão (SIMÕES MV, et al., 2018).

Em relação a forma indeterminada, esta é caracterizada com evidência parasitológica e/ou sorológica de infecção crônica na ausência de sintomas e sinais clínicos típicos da entidade nosológica, a qual pode perdurar 30 a 40 anos, apresentando exames complementares como eletrocardiograma e radiografia de tórax sem alterações, assim como sem sinais de comprometimento de esôfago e cólon observados por exames radiológicos. A forma crônica determinada cardíaca representa a forma clássica da cardiomiopatia crônica da 
doença de Chagas com sinais e sintomas que tem como base quatro síndromes que podem se apresentar concomitantemente no mesmo paciente, são elas: manifestações anginosas, arritmias, insuficiência cardíaca e tromboembolismo (SIMÕES MV, et al., 2018).

A forma cardíaca pode ser ainda classificada em estágios clínicos ( $A, B, C$ e D) dependendo da progressão do envolvimento cardíaco. O estágio $A$ é a forma indeterminada. $O$ estágio $B$ contempla os pacientes sem clínica de insuficiência cardíaca, porém, que podem apresentar alterações sugestivas de cardiopatia estrutural nos exames complementares, sendo divididos em duas categorias: B1, com alterações eletrocardiográficas, tais como distúrbio de condução ou arritmias, sem disfunção ventricular global ao eletrocardiograma, mas com a possibilidade de existir anormalidades da contratilidade regional ao exame ecocardiográfico; e B2, que cursa com disfunção ventricular global e fração de ejeção de ventrículo esquerdo reduzida (ANDRADE JA, et al., 2011).

No estágio $\mathrm{C}$, soma-se à disfunção ventricular os sintomas prévios ou atuais de insuficiência cardíaca. Já no estágio $\mathrm{D}$, a sintomatologia de insuficiência cardíaca dos pacientes manifesta-se em repouso, classe funcional IV segundo a classificação da New York Heart Association (NYHA) sendo refratária ao tratamento otimizado, com necessidade de terapêuticas de maior complexidade (ANDRADE JA, et al., 2011).

A história natural da cardiopatia chagásica acomete os três folhetos cardíacos e o sistema de condução. Há destruição de fibras miocárdicas pelo processo inflamatório crônico e a sua substituição por tecido fibroso, levando ao remodelamento dos ventrículos com perda gradativa dos elementos contráteis. As manifestações cardíacas geralmente se iniciam com alterações no sistema de condução mais frequentemente com bloqueio completo de ramo direito ou bloqueio fascicular anterior esquerdo e alteração de mobilidade segmentar do ventrículo esquerdo. Posteriormente, as manifestações incluem extra-sístoles e taquicardias ventriculares, disfunção e/ou doença do nó sinoatrial, bloqueios atrioventriculares, fenômenos tromboembólicos e cardiomiopatia em fase dilatada com insuficiência cardíaca congestiva (DIAS JC, et al., 2016; PEDROSA RC, et al., 2013; ANDRADE JA, at al., 2011).

Esses acometimentos levam a uma taxa de mortalidade de 4\%, como relata Silva HC, et al. (2013), sendo que dentre essas mortes, $55 \%$ a $65 \%$ são súbitas, $25 \%$ a $30 \%$ por insuficiência cardíaca refratária, e $10 \%$ a $15 \%$ por eventos tromboembólicos.

Dentre as opções medicamentosas para o tratamento da Doença de Chagas, o benznidazol e o nifurtimox são alternativas possíveis na abordagem clínica das fases aguda e crônica da doença, com menor impacto nas taxas de cura e nas lesões cardíacas já estabelecidas nesta última fase citada, agindo predominantemente sobre o parasita. (PAHO, 2019)

Diante do exposto, o presente estudo tem por finalidade analisar a evolução da cardiomiopatia chagásica em pacientes adultos jovens, identificando complicações e abordagens diagnósticas e terapêuticas mais atuais.

\section{MÉTODOS}

Trata-se de uma revisão integrativa da literatura elaborado como um método organizado, como referido por Mendes KDS, et al. (2008), que reúne e sintetiza resultados de pesquisas sobre um determinado assunto.

O estudo foi norteado pelas etapas preconizadas por Ribeiro OMPL, et al. (2016), constituída por: formulação da questão para a elaboração da revisão integrativa da literatura, especificação dos métodos de seleção dos estudos, procedimento de extração dos dados, análise e avaliação dos estudos incluídos na revisão integrativa da literatura, extração dos dados e apresentação da revisão/síntese do conhecimento produzido e publicado.

A presente revisão teve como questão norteadora: quais são as complicações e abordagens terapêuticas da cardiomiopatia chagásica em pacientes jovens? Na escolha dos estudos a serem incluídos na pesquisa recorremos à estratégia de População/participantes, Intervenções, Comparação/controle e Outcomes/desfechos (PICO) como mostra o Quadro 1. 
Quadro 1 - Estratégia PICO na escolha dos estudos incluídos na pesquisa nos últimos 10 anos.

\begin{tabular}{|c|c|c|}
\hline P & Participantes & Pacientes jovens com cardiomiopatia chagásica \\
\hline I & Intervenção & $\begin{array}{r}\text { Intervenções diagnósticas ou terapêuticas no contexto de } \\
\text { complicações cardiológicas da doença }\end{array}$ \\
\hline C & Contexto & Hospitalizados ou em tratamento ambulatorial \\
\hline O & Resultados/Outcomes & Resultados \\
\hline
\end{tabular}

Fonte: Morais AFP, et al., 2020.

A coleta de dados ocorreu no período de janeiro a maio de 2020 nas bases de dados: Public Medline ou Publisher Medline (PubMed), Literatura Latino-Americana e do Caribe em Ciências da Saúde (LILACS), Scientific Electronic Library Online (SCIELO), Science.gov e Medline.

Os critérios de exclusão foram: artigos que não abordaram especificamente sobre complicações ou pacientes chagásicos ou pacientes jovens. Definiram-se como critérios de inclusão: estudos publicados no espaço de tempo 2010-2020, acesso permitido em texto integral, nos idiomas inglês, português e espanhol, e cujo título e/ou resumo fizessem referência à temática da cardiomiopatia chagásica em adultos jovens com referência às complicações da doença, sendo colocados os seguintes descritores: Cardiomiopatia chagásica; e complicações (Chagasic cardiomyopathy; and complication).

Da pesquisa nas bases de dados, resultou a identificação de 184 artigos. Num primeiro momento, foi realizada uma leitura crítica e reflexiva dos títulos e dos resumos encontrados. Posteriormente, após a aplicação dos critérios de inclusão e exclusão, e a avaliação da qualidade metodológica, estabeleceu-se uma amostra de 21 artigos. Num segundo momento, procedeu-se a uma análise criteriosa dos artigos selecionados, extraindo dos mesmos as evidências relativas aos estudos que falavam de cardiopatia chagásica em pacientes jovens e/ou de suas complicações, resultando em nove artigos como base para discussão, como demonstrado no fluxograma a seguir (Figura 1).

Figura 1 - Fluxograma detalhando coleta de dados e processo de análise criteriosa dos artigos selecionados.

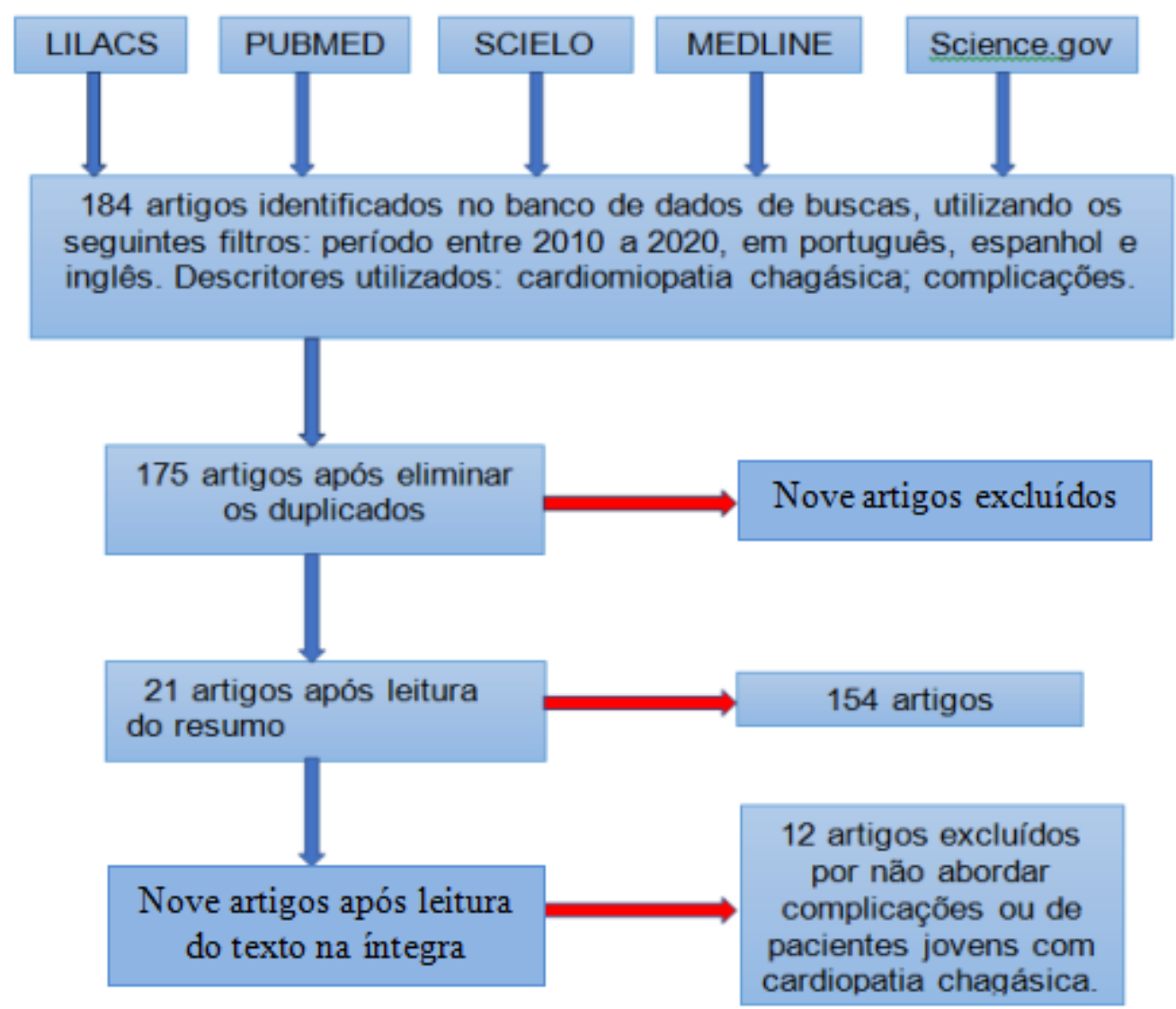

Fonte: Morais AFP, et al., 2020. 
Com o intuito de sistematizar a informação dos artigos, os dados extraídos dos estudos foram compilados de forma descritiva e os artigos foram sistematizados por uma classificação de evidências caracterizados de forma hierárquica, dependendo da abordagem metodológica adotada, e ambos foram organizados em uma tabela previamente elaborada, o que facilitou a identificação e a reformulação das categorizações temáticas (SOUZA MT, et al., 2010).

$\mathrm{Na}$ análise das evidências dos artigos selecionados, utilizou-se a classificação descrita por Melnyk BM e Fineout-Overholt E (2005), composta por sete níveis: nível um: evidências de revisão sistemática ou metanálise ou diretrizes clínicas baseadas em revisões sistemáticas de ensaios clínicos randomizados controlados relevantes; nível dois: evidências derivadas de um ensaio clínico randomizado controlado relevante; nível três: evidências obtidas de ensaios clínicos sem randomização; nível quatro: evidências de estudo de caso controle e coorte; nível cinco: evidências de estudos descritivos e qualitativos em revisão sistemática; nível seis: evidências de um único estudo descritivo ou qualitativo e o nível sete: evidências de opiniões de relatórios de comitês de especialistas e/ou autoridades.

As análises metodológicas foram realizadas por três pesquisadores, de forma independente, e no caso de divergência de opiniões, houve a avaliação de um quarto pesquisador.

Por fim, realizou-se a análise por meio da síntese das publicações selecionadas de maneira descritiva, com ênfase no conhecimento produzido acerca da pergunta norteadora, proporcionando uma crítica dos resultados e a qualidade das evidências.

\section{RESULTADOS}

Esta revisão procurou analisar publicações científicas relacionadas à temática da cardiomiopatia chagásica em jovens com referência às complicações da doença. A finalidade foi avaliar e sintetizar os conhecimentos sobre a evolução dessa entidade nosológica em pacientes jovens, suas principais complicações e a frequência com que ocorrem, assim como as abordagens diagnósticas e terapêuticas que constam na literatura mais atual (Quadro 2). 
Quadro 2 - Revisão integrativa no PubMed, LILACS, SCIELO, MEDLINE e Sciense.gov.

\begin{tabular}{|c|c|c|c|}
\hline Procedência & Referência & Considerações/temática & Nível de evidência \\
\hline PubMed & Lassen O, et al. (2013) & $\begin{array}{l}\text { Descreve uma pior evolução da miocardiopatia chagásica em mulheres jovens } \\
\text { independentes de outros fatores de risco cardiovascular. }\end{array}$ & Nível 3 \\
\hline PubMed & Tobar IB, et al. (2011) & $\begin{array}{l}\text { Diferencia pacientes soropositivos de pacientes soronegativos para o Trypanosoma } \\
\text { Cruzi inferindo uma correlação significativa dos soropositivos e a presença de uma } \\
\text { miocardite moderada ou grave. }\end{array}$ & Nível 4 \\
\hline Science.gov & Woudstra L, et al. (2018) & $\begin{array}{l}\text { Descreve o papel da regulação da microvasculatura miocárdica e correlaciona a } \\
\text { redução da perfusão miocárdica e a redução de fluxo de reserva coronariano com a } \\
\text { severidade da miocardiopatia chagásica aguda e crônica e suas complicações } \\
\text { cardíacas }\end{array}$ & Nível 5 \\
\hline LILACS & Vinajá D e Aché A (2012) & $\begin{array}{l}\text { Analisa as principais alterações eletrocardiográficas de pacientes com cardiomiopatia } \\
\text { chagásica, notando-se predomínio de bloqueios de ramo direito e fibrilação atrial. }\end{array}$ & Nível 4 \\
\hline Science.gov & Hidron Al, et al. (2010) & $\begin{array}{l}\text { Relaciona as alterações clínica e ecocardiográfica com a progressão da cardiomiopatia } \\
\text { chagásica e os fatores prognósticos }\end{array}$ & Nível 4 \\
\hline Science.gov & Torreão JA, et al. (2015) & $\begin{array}{l}\text { Correlaciona a presença, a localização e a quantificação da fibrose miocárdica } \\
\text { identificada pela Ressonância Magnética de Miocárdio com a gravidade da doença }\end{array}$ & Nível 6 \\
\hline LILACS & Almeida DR (2016) & $\begin{array}{l}\text { Relata que os resultados do transplante cardíaco na doença de Chagas são altamente } \\
\text { satisfatórios e com evolução pós-transplante a médio e longo prazo semelhante aos } \\
\text { resultados do transplante cardíaco em pacientes não chagásicos. }\end{array}$ & Nível 2 \\
\hline LILACS & Vilas-Boas F, et al. (2011) & $\begin{array}{l}\text { Avalia em seres humanos a segurança e a eficácia do transplante de célula da medula } \\
\text { óssea no miocárdio de pacientes com insuficiência cardíaca devido à cardiomiopatia } \\
\text { chagásica. }\end{array}$ & Nível 2 \\
\hline SCIELO & Giménez LJ, et al. (2019) & $\begin{array}{l}\text { Além de realizar uma revisão no tratamento dos pacientes com cardiomiopatia } \\
\text { chagásica com fração de ejeção reduzida, utilizando as medicações habituais e doses } \\
\text { otimizadas, mostra que esses pacientes são mais jovens e têm menos comorbidades } \\
\text { do que os cardiopatas de outras etiologias e continuam tendo uma pior qualidade de } \\
\text { vida e maior mortalidade. }\end{array}$ & Nível 5 \\
\hline
\end{tabular}

Fonte: Morais AFP, et al., 2020. 


\section{DISCUSSÃO}

O artigo de Lassen O, et al. (2013) aborda as condições cardíacas em mulheres jovens com a Doença de Chagas. Nessas pacientes os níveis de estrogênio não protegem contra o processo patológico gerado pela infecção do T. Cruzi, pois elas desenvolveram dano miocárdico antes da menopausa. Além disso, observouse a agressividade da doença evidenciada pela diminuição grave da fração de ejeção (menor que $40 \%$ ) e pela dilatação das cavidades cardíacas grau III, no ecocardiograma. Apesar de demonstrar que os fatores hormonais não apresentam proteção como relatado em outras doenças cardíacas, o artigo não demonstra como ocorre esse dano e nem menciona a razão do mecanismo de agressão ser tão precoce, sendo necessárias novas pesquisas que abordem sobre 0 assunto.

A presença de anticorpos anti $T$. Cruzi em pacientes com insuficiência cardíaca descompensada, analisada por Tobar IB, et al. (2011), sugere associação com desenvolvimento de cardiomiopatia chagásica crônica e a soropositividade deve ser considerada um fator de risco para o desenvolvimento de insuficiência cardíaca. Ressalta-se que a cardiomiopatia chagásica crônica é caracterizada por bloqueio de ramo direito associado com hemibloqueio anterior esquerdo, arritmias ventriculares e atriais, bradiarritmias, disfunção biventricual, aneurisma ventricular apical, eventos trombóticos e morte súbita.

Além dessas complicações citadas, notou-se que pacientes com doença de Chagas têm um prognóstico pior do que pacientes com insuficiência cardíaca resultante de outras etiologias. O pior prognóstico está associado com a presença de QRS prolongado, taquicardia ventricular, fração de ejeção baixa, bradicardia e diminuição da pressão sistólica (TOBAR IB, et al., 2011).

Diante de tais sinais, embasado no artigo de Tobar IB, et al. (2011), nota-se a necessidade de realizar a sorologia nos primeiros sinais de alteração eletrocardiográfica, na tentativa de minimizar as complicações e melhorar o prognóstico, visto que é um exame de fácil acesso e de fundamental importância na avaliação da doença. Em relação ao atendimento médico geral no país, mostra-se necessário reforçar, nos protocolos de arritmias e insuficiência cardíaca, assim como em nível primário, a importância da solicitação precoce da sorologia para Doença de Chagas.

O artigo de Woudstra L, et al. (2018) aponta para a patogênese das alterações encontradas na cardiomiopatia chagásica crônica. Nesta patogênese, tanto a microvasculatura intramiocárdica quanto as artérias coronárias epicárdicas desempenhariam importante papel na progressão da doença, pois apresentam função de barreira de proteção para impedir a entrada dos parasitas nos tecidos cardíacos, porém, também possuem endotélio passível de infecção por estes patógenos e de ataque pela resposta imune pós-infecção.

O resultado é o surgimento de alterações estruturais progressivas que, posteriormente, irão repercutir em agravamento do dano miocárdico e da função cardíaca como um todo. Acredita-se que parte das alterações esteja relacionada com proliferação das células endoteliais, com aumento do calibre dos capilares. Concomitantemente, nota-se relação com o aumento da permeabilidade desses vasos, progressão de trombogênese e aterosclerose, alterações da função vasomotora coronariana e prejuízo da perfusão cardíaca (WOUDSTR L, et al., 2018). A patogênese explicada no artigo aborda alterações em longo prazo, progressivas, que não explicariam as alterações mais graves e em tempo menos prolongado, que podem ocorrer em pacientes mais jovens, sendo necessárias novas pesquisas que abordem sobre 0 assunto.

Em estudo epidemiológico de Vinajá D e Ache A (2012), notou-se que, em relação às alterações eletrocardiográficas, os distúrbios de condução mais frequentes foram bloqueio de ramo (53\%), predominantemente direito, e fibrilação atrial (a mais frequente), com $55,3 \%$. Ressalta-se que em comparação com outros estudos realizados pelo mundo e citados no estudo em questão, observou-se que a alteração eletrocardiográfica mais frequente foi bloqueio de ramo na maioria dos casos, porém, em outros, foi a fibrilação atrial, apesar de não ser tão frequente. Observa-se que em todos os estudos epidemiológicos, em pacientes com cardiomiopatia chagásica, há algum distúrbio de condução, demonstrando suas complicações cardíacas.

Tais estudos epidemiológicos, citados no artigo de Vinajá D e Ache A (2012), são de grande importância para a identificação da cardiomiopatia chagásica crônica, sendo utilizados na prática clínica para suspeita e 
investigação da doença. Entretanto, nota-se que mais estudos sobre mortalidade e complicações em pacientes jovens precisam ser realizados e difundidos no ensino médico, pois, ainda existem profissionais da saúde que não sabem reconhecer essas características da doença.

O artigo de Hidron A, et al. (2010) cita o desenvolvimento de alterações no ecocardiograma de paciente com doença de Chagas crônica, atentando para a disfunção diastólica, que geralmente precede a disfunção sistólica, permitindo uma detecção precoce do envolvimento cardíaco nesses pacientes. Além disso, o artigo cita o desenvolvimento de aneurismas apicais (em 8,5 a $55 \%$ dos pacientes), anormalidades na contratilidade de segmentos do ventrículo esquerdo, principalmente da parede póstero-inferior, e função sistólica reduzida desta mesma câmara.

A importância das evidências ecocardiográficas de disfunção do ventrículo esquerdo está no fato de representarem o mais importante preditor independente de óbito. Outros indicadores prognósticos seriam classificação da NYHA avançada (classe III/IV), cardiomegalia e taquicardia ventricular não sustentada no holter de 24 horas. $O$ autor cita também que a progressão para os estados mais avançados da doença ocorre em cerca de $10 \%$ dos pacientes em um período de três a 10 anos de seguimento, com expectativa de vida menor que $30 \%$ em cinco anos para aqueles com cardiomiopatia avançada e sintomática (HIDRON A et al., 2010).

Apesar dos benefícios de tais exames citados no artigo de Hidron A, et al. (2010), há que se ressaltar que eles não se encontram disponíveis em muitos serviços médicos, além da necessidade de profissionais qualificados e treinados para manejar esses aparelhos, em especial os exames de avaliação da função ventricular como ecocardiograma e de arritmias como o Holter.

O estudo de Torreão JA, et al. (2015) analisou as alterações visíveis através da ressonância magnética computadorizada (CMR) comparando 54 pacientes de faixa etária similar com doença de Chagas em três fases clínicas distintas: fase indeterminada (IND), fase cardíaca crônica sem disfunção do ventrículo esquerdo (CPND) e cardíaca crônica com disfunção desta câmara (CPD).

Concluiu-se a que a CMR permite avaliar a severidade da cardiomiopatia chagásica, identificando a presença, a localização e a quantidade de fibrose miocárdica, além de hipersinal do miocárdio em imagens ponderadas em T2 (T2W) e realce precoce do gadolínio no miocárdio em imagens ponderadas em T1 (MEGE). Notou-se presença de T2W e MEGE em todas as fases da doença, com importante correlação de severidade, classe funcional de NYHA, função ventricular esquerda e grau de fibrose (TORREÃO JA, et al., 2015).

Assim, embasado no artigo de Torreão JA, et al. (2015), observa-se que a CMR pode representar uma importante ferramenta no seguimento desses pacientes, entretanto, é um exame caro, pouco acessível na maioria dos serviços, sendo inviável financeiramente para o seguimento desses pacientes, além de exigir um profissional treinado para manejar o aparelho. Como ferramentas de seguimento o eletrocardiograma e o ecocardiograma ainda se mostram mais disponíveis em comparação à ressonância magnética.

Quanto ao transplante cardíaco, opção terapêutica abordada pelo artigo de Almeida DR (2016), observase que a experiência tida no Brasil, por mais que ocorram taxas elevadas de reativação da infecção pelo $T$. Cruzi em consequência de um estado de imunocomprometimento, os resultados obtidos pelo transplante cardíaco na miocardiopatia chagásica possuem evolução pós-transplante satisfatória semelhante aos de pacientes não chagásicos que também foram submetidos a esse procedimento. Com esse resultado, podese colocar o transplante cardíaco como uma opção terapêutica relevante em pacientes chagásicos com arritmias. Apesar de ser um tratamento promissor, a sua disponibilidade é limitada para muitos pacientes em decorrência de contraindicações e dificuldades de acesso a centros de transplantes.

O relato da primeira experiência humana acerca do transplante de células da medula óssea realizado em pacientes com insuficiência cardíaca causada pela doença chagásica foi efetuado por Vilas-Boas $\mathrm{F}$, et al. (2011) e demonstra ser efetivo e viável. Essa terapia celular foi feita por meio de injeção intracoronariana de células mononucleares provenientes de medula óssea autóloga e foi considerada segura, uma vez que o 
procedimento não teve consequências como dano ao miocárdio e deterioração da função cardíaca bem como arritmias, e pôde-se obter um aumento relativo de $20 \%$ da fração de ejeção do ventrículo esquerdo (FEVE) em pacientes que a tinham significativamente diminuída.

A terapia celular, relatada no artigo de Vilas-Boas F, et al. (2011), apesar de ter sido considerada segura, ainda é um tratamento muito novo, com pouca experiência, sendo necessários maiores estudos, investimentos e maior número de pacientes com a doença recebendo o tratamento, para posteriormente, entrar em protocolos e ser uma opção de tratamento eficaz e alternativa. Além de ser uma terapia nova, ainda é pouco acessível em países em desenvolvimento devido ao elevado custo.

No artigo de Giménez LJ, et al. (2019) destacam-se algumas indicações para o transplante cardíaco, como em pacientes com arritmias que causam episódios de morte súbita, apesar do tratamento com antiarrítmicos e dispositivos implantáveis, e em pacientes com IC (Insuficiência Cardíaca) Classe Funcional (CF) III-IV (NYHA), apesar do tratamento médico otimizado e não passível de outro tratamento alternativo mais simples. Dentre as contraindicações, as absolutas merecem destaques, sendo: doenças limitantes à vida, insuficiência hepato-renal significativa, amiloidose e incapacidade grave que impossibilita a reabilitação.

$\mathrm{O}$ artigo ainda ressalta o tratamento com benznidazol em pacientes com processos mais avançados de doença cardíaca. Esse tratamento etiológico não demonstrou ser eficaz na melhoria do curso da doença, conforme avaliado no estudo BENEFIT, que apesar da negativação das sorologias para $T$. Cruzi com o tratamento do benznidazol, não houve diminuição de mortalidade e desfechos clínicos (MORILLO CA, et al., 2015; GIMÉNEZ J, et al., 2019).

O tratamento é indicado apenas na miocardite chagásica aguda ou na cardiopatia chagásica crônica, produto de reinfecção endógena, em pacientes transplantados, com Síndrome da Imunodeficiência Adquirida (AIDS) ou imunossuprimida ou por outra causa e receptor chagásico no transplante de órgãos como profilaxia (Classe I / nível de evidência C) (GIMÉNEZ LJ, et al., 2019). Tal estudo demonstra que as indicações de benznidazol são restritas e que o uso sem indicação além de não ser eficaz, pode atrasar um tratamento mais adequado e piorar prognóstico. Apesar do estudo BENEFIT, poucos profissionais da saúde têm acesso a essas informações, sendo necessário que essas indicações sejam mais divulgadas.

\section{CONSIDERAÇÕES FINAIS}

As complicações encontradas são caracterizadas por: bloqueio de ramo direito associado com hemibloqueio anterior esquerdo, arritmias ventriculares e atriais, bradiarritmias, disfunção biventricular, aneurisma ventricular apical, eventos trombóticos e morte súbita, estando o pior prognóstico associado com a presença de QRS alargado, taquicardia ventricular, fração de ejeção baixa, bradicardia e diminuição da pressão sistólica. Dentre as alterações ecocardiogáficas, nota-se a disfunção diastólica precedendo a disfunção sistólica. Conclui-se que os exames eletrocardiograma, ecocardiograma e ressonância magnética ajudam a identificar a evolução da cardiopatia e indicar tratamento precoce. A escassez de estudos mostra a necessidade de desenvolver pesquisas e terapias que melhorem a sobrevida desses pacientes.

\section{REFERÊNCIAS}

1. ALMEIDA DR. Transplante cardíaco na doença de Chagas / Heart transplantation in Chagas' disease. Rev. Soc. Cardiol., 2016; 26(4): 266-71.

2. ANDRADE JA, et al. Sociedade Brasileira de Cardiologia. I Diretriz Latino Americana para o Diagnóstico e Tratamento da Cardiopatia Chagásica. Arq Bras Cardiol., 2011; 97(2 supl.3): 1-48.

3. BOCCHI EA, et al. Novas abordagens terapêuticas na doença de chagas: terapia celular, anticorpos, aférese. Rev Soc Cardiol., 2016; 26(4): 261-5.

4. DIAS JC, et al. II Consenso Brasileiro em Doença de Chagas. Epidemiol. Serv. Saúde, 2016; 25(núm. esp.): 7-86.

5. GIMÉNEZ LJ, et al. Actualización de diagnóstico y tratamento de la insuficiencia cardíaca de etiologia chagásica. Insuf Card. Consenso de enfermedad de chagas, 2019; 14 (3): 96-110.

6. HIDRON Al, et al. Chagas cardiomyopathy in the context of the chronic disease transition. PLOS Neglected Tropical Diseases, 2010; 4(5).

7. JUNIOR ASM, et al. Chronic Chagas Cardiomyopathy Patients and Resynchronization Therapy: a Survival Analysis. Braz J Cardiovasc Surg., 2018; 33 (1):82-8. 
8. LASSEN O, et al. Cardiac condition in young chagasic women. Journal of Medicine and Life, 2013; 6(2): 185-188.

9. MARTINS-MELO FR, et al. Prevalence of Chagas disease in Brazil: a systematic review and meta-analysis. Acta Trop., 2014; 130: 167-74.

10. MELNYK BM, FINEOUT-OVERHOLT E. Making the case for evidence-based practice. In: Melnyk BM, FineoutOverholt E. Evidence-based practice in nursing \& healthcare. A guide to best practice. Philadelphia: Lippincot Williams \& Wilkins, 2005; p. 3-24.

11. MENDES KDS, et al. Revisão integrativa: método de pesquisa para a incorporação de evidências na saúde e na enfermagem. Texto Context - Enferm. 2008; 17(4): 758-64.

12. MORILLO CA, et al. BENEFIT Investigators. Randomized Trial of Benznidazole for Chronic Chagas' Cardiomyopathy N Engl J Med., 2015; 373: 1295-1306.

13. PAHO. PAN AMERICAN HEALTH ORGANIZATION. Guidelines for the diagnosis and treatment of Chagas disease. Washington, D.C.; 2019.

14. PEDROSA RC, et al. Cardiopatia Chagasica. Revista da Sociedade de Cardiologia do rio de Janeiro. Agosto, 2013 Disponível em https://socerj.org.br/cardiopatia-chagasica/.

15. RIBEIRO OMPL, et al. Modelos de prática profissional de enfermagem: revisão integrativa da literatura. Revista de Enfermagem Referência, 2016; Série IV(no10): 125-133.

16. SCHIMIDT A, et al. Epidemiologia no século XXI e aspectos clínicos da doença de chagas crônica. Rev Soc Cardiol., 2016; 26(4): 230-3.

17. SILVA HC, et al. Avaliação Clínica na atenção Primária e Infectologia dos Pacientes com Doença de Chagas na forma Crônica. Revista Baiana de Saúde Pública, 2013; 37(Suplemento 1): 7-21.

18. SIMÕES MV, et al. Cardiomiopatia da doença de Chagas. International Journal of Cardiovascular Sciences, $2018 ; 31$ (2): 173-189.

19. SOUZA MT, et al. Revisão integrativa: o que é e como fazer. Einstein, 2010; 8 (1Pt1): 102-6

20. TOBAR IB, et al. Prevalence of Trypanosoma cruzi antibodies and inflammatory markers in uncompensated heart failure. Revista da Sociedade Brasileira de Medicina Tropical, 2011; 44(6): 691-696.

21. TORREÃO JA, et al. Myocardial tissue characterization in Chagas' heart disease by cardiovascular magnetic resonance. J Cardiovasc Magn Reson, 2015; 17:97.

22. VILAS-BOAS F, et al. Transplante de células da medula óssea na insuficiência cardíaca chagásica: relato da primeira experiência humana. Arq. Bras. Cardiol.. 2011; 96.

23. VINAJÁ D, ACHÉ A. Alteraciones Electrocardiográficas em pacientes com Enfermedad de Chagas. Hospital José Rangel de Villa de Cura. 1998-2008. Revista del Instituto Nacional de Higiene Rafael Rangel, 2012; 43(2).

24. WOUDSTRA L, et al. Infectious myocarditis: the role of the cardiac vasculature. Heart Fail Rev., 2018; 23(4): 583-95. 